\title{
Appropriate Assembly Instruction Modes: Factors to Consider
}

\author{
Nathanaël KUIPERS ${ }^{1}$, Ari KOLBEINSSON and Peter THORVALD \\ Department of Engineering, University of Skövde, Högskolevägen 1, 54125 Skövde, \\ Sweden
}

\begin{abstract}
Presented is a literature study into the importance of how information in assembly instructions in manual assembly is presented, more specifically how various factors such as the complexity of the assembly itself, the mental and physical workload of the worker, as well as the experience and skill level of the worker affect the requirements for information presentation. The requirements made by Industry 4.0 on flexibility in production lines and an increased number of variants produced causes increased demands on workers, which leads to more cognitive demands being made on assembly workers. Studies exist around assembly instruction modes, but have in many cases ignored factors such as worker skill level, mental workload, and task complexity and how these affect the requirements for information presentation, which is a major contribution of this study. The findings are that no single solution fits all requirements, but that the aforementioned factors should be taken into account.
\end{abstract}

Keywords. Assembly Process, Assembly Instructions, Assembly Guidance, Design for Assembly, Augmented Reality, Poka-Yoke, Cognition

\section{Introduction}

Manufacturers have been attempting to increase their performance throughout history, with multiple industrial revolutions offering dramatic increases some aspects of production capability. The focus of these production improvements has historically focused on making production lines more effective and efficient, leading to mass production of identical objects. The end of the twentieth century introduced a new consumer demand that coincided with new capabilities in managing production lines; namely customised products. Customising products during production requires communicating any differences from the base product to the workers creating these items, and leads to a high number of alternative versions of the same product. When to make a specific variant must be communicated at the correct time, to the correct person, and care must be taken to support the worker in performing the assembly activity correctly, as an increase in the number of variables to take into consideration leads to a similar increase in the risk of manufacturing errors [1]

The increased demand on manufacturing workers due to the increase in customisation and higher levels of optimisation made possible by Industry 4.0 enabled manufacturing means that the digital transformation offered by Industry 4.0 must also aid workers in filtering and processing information, reduce their cognitive load and

\footnotetext{
${ }^{1}$ Corresponding Author. Nathanael.kuipers@his.se
} 
supporting the worker in more ways, which can lead to higher worker satisfaction and higher performance [2].

One such domain where workers can be supported is by introducing assembly tasks and assembly instructions that take into account worker cognitive capabilities and limitations. As assembly tasks and worker capabilities can vary it is entirely possible that no one optimal way of presenting assembly instructions exists for assembly tasks of varying complexity and difficulty, for workers with varying experience levels, as well as under differing workloads. Understanding how to present instructions that are appropriate for a particular assembly activity and worker is what will be explored here.

\section{Background}

Instructions can generally be seen as a form of information which someone has to act upon. Instructions can be given in different formats; auditory, tactile and visual, where the receiver uses senses like hearing, touch and sight. [3]. Independent of what mode of presentation is being used it is important that the receiver decodes correctly what the sender is requesting be done and acts accordingly. Interpreted to a manual assembly line this might be; the worker receives instructions on what to do, has to decode and interpret what is asked, and then execute the task. The process then repeats itself where the worker either receives similar or a new set of instructions. The process between receiving one instruction to the next can be referred to as an assembly cycle. A schematic overview of a cycle can be seen in figure 1 .

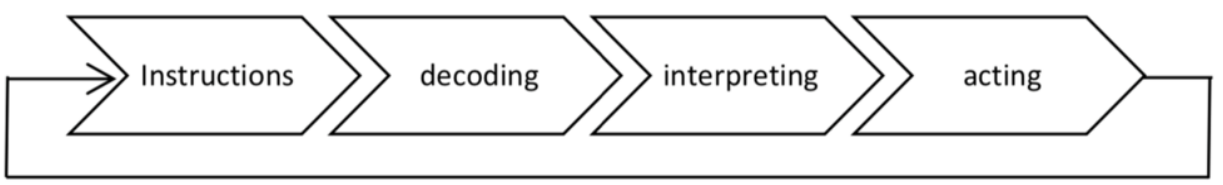

Figure 1. The process of an assembly cycle.

One central aim is to get the highest possible overall performance during the whole assembly process which means that the assembly cycle has to be both effective and efficient. The Lean philosophy defines effectiveness as doing the right thing, and efficiency as doing things the right way. Effectiveness is connected to quality and reducing errors, efficiency to reducing time and effort [4].

Instructions play an important role in both effectiveness and efficiency during the assembly process. Instructions have to be well documented, clear and concise, easy to use by the intended user, but most important is that the instructions are interpreted in the exact same way by different receivers to minimise the risk of confusion and errors as well as to guarantee quality $[5,6]$. However the quality of the instructions is not the only factor that can lead to confusion and errors. The instructions can be crystal clear, but if the assembly operation itself is causing problems, the worker might still question if either the instructions are incorrect or if they are misinterpreted, even though there is a chance that neither is true. In such situations the worker would like to know as soon as possible and with minimum effort what is causing this discrepancy.

The question being explored is: In what way should instructions be provided to the manual assembly worker to maximise assembly performance? 
This question is too large to explore on its own, and is thus split into four subquestions:

RQ1: What is the task the assembly worker needs to perform?

$R Q 2:$ Which information needs to be conveyed to the assembly worker to perform the task?

RQ3: Which information is essential to maximise assembly performance?

RQ4: What format or mode is most effective in conveying essential information?

The aim of the study is to analyse and assess the factors that play a role in the effectiveness of instructions in manual assembly operations and to predict how to deliver instructions in the most effective way for certain manual assembly operations. The identified factors of influence are:

- The difficulty of the respective assembly operation

- The information that needs to be conveyed through instructions

- The format or mode that the information is delivered in

- The experience and skills of the manual assembly worker

\section{Method}

A snowballing approach [7] was used for identifying relevant research, followed by scanning and sorting based on relevance of abstracts. Initial keywords were searched, and the identified articles were mined for related keywords and key concepts. After the initial search new search strings were applied, using terms that had been identified during the initial search as being related to the word instruction. Examples of this are: task, guideline, guidance, or (visual) aid. This led to a few more articles of interest but not to significant new discoveries. Search tools used included Google Scholar, WorldCat, and ScienceDirect. The structure and method of the literature review, including some of the core search terms, can be seen in figure 2 .

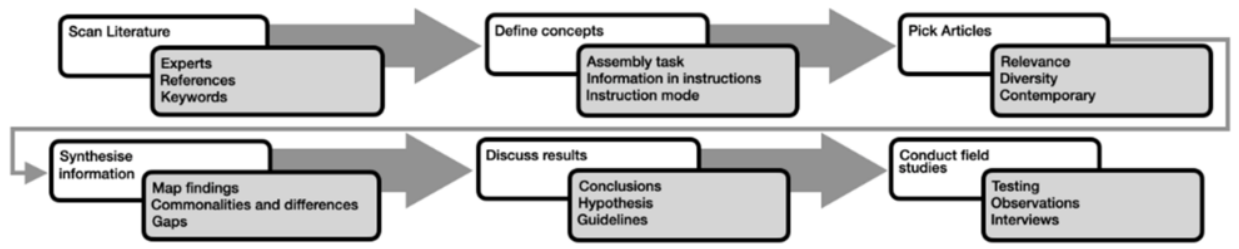

Figure 2. Used method consisting of an information gathering phase (top row) and a developing phase (bottom row).

\subsection{Identified themes}

Analysing the articles led to three themes being identified. Manual assembly task led to the field of design for manufacturing and assembly (DFMA) for which detailed literature is already available. Manual assembly instruction or guidance led to a) research within cognitive load for assembly workers when presenting instructions, and b) the mode of presentations, where e.g. AR/VR presentation of instructions is compared to more traditional paper instructions.

This, in turn, allowed more defined searches where search terms to do with cognition or variations on augmented reality were combined with the manual assembly instruction search term, which resulted in five more identified papers of high relevance. 
Although around half a million articles came up in an initial search, refining the search terms and identifying relevant articles reduced the total number of relevant articles to 31, which can be argued to be a low number. Li et al. [8] similarly came to the conclusion that there is comparatively little contemporary research or clear guidelines on (digital) assembly instructions.

\section{Results of the literature review}

The literature study suggests that there is at the moment no consensus on what can be considered best practice when it comes to manual assembly instructions. An explanation could be that the conducted tests all had different characteristics with different test parameters. Examples of this is how Alkan [9] assigns complexity factors to components, liaisons, and topology of the product, while DFA based approaches work with retrieving, handling, positioning, and joining. The different ways of defining the work being performed can thus complicate meta-analyses.

Many of the reviewed articles tend to either take into consideration the abovementioned factors to do with the product $[10,11$, or complexity based factors $[12,13]$.

In addition to this, it was found that the reviewed papers have differing strengths. Boothroyd et al. [14] contain robust and detailed data from practical experiments, while Agrawala et al. [12] thoroughly examine how paper instructions can be presented.

Even though there are some gaps after analysing, comparing, and mapping the input from the different research fields, a certain commonality and overlap was identified between issues and themes. These common aspects have been collected and can be seen below.

\subsection{Commonalities between identified themes}

The three identified themes, DFA and the assembly task, interpreting and decoding instructions, and Different instruction modes, were found to share some commonalities. Analysing articles within the themes of articles led to the following areas being identified where existing research was largely in agreement regarding manual assembly and instructions:

1. Performance is measured in efficiency (time) and effectiveness (number of errors).

2. The assembly process can be divided into retrieving a part, handling a part, positioning a part, and joining a part.

3. Operations can be of different complexity, depending on the complexity of the part, the liaison and the intricateness of the product, leading to different levels of mental effort.

4. A strong correlation exists between increasing complexity of the task and decreasing performance (increase in time and errors).

5. The assembly process has to be channeled and explained through instructions: what has to go where and how.

6. A set of instructions has to be decoded and interpreted by the worker, and then acted upon, which contributes to mental load.

7. Mental load and mental effort are both factors in an assembly cycle, and are additive.

8. Tasks are divided into a series of subtasks (actions). 
9. Beginners perform better with step-by-step instruction based information, while trained workers are prone to err more using the same information, and prefer a more holistic approach to instructions.

10. Conventional (paper-based) step-by-step instructions still perform well in comparison with modern technologies.

11. Instruction presentation through current head mounted display (HMD) products and technologies were not seen as leading to higher performance. The results further indicate that current HMD systems increase the mental load experienced by the user, even though many users find it an interesting medium to use.

Table 1:Which article contains each of the 11 identified issues. Numbers correspond to the above list, letters correspond to A) Assembly task, B) Information in instructions, and C) Instruction mode.

\begin{tabular}{|c|c|c|c|c|c|c|c|c|c|c|c|c|}
\hline & Theme & 1 & 2 & 3 & 4 & 5 & 6 & 7 & 8 & 9 & 10 & 11 \\
\hline Agrawala et al. (2003) & B & $\mathrm{X}$ & & & & $\mathrm{x}$ & & & $\mathrm{x}$ & $\mathrm{x}$ & $\mathrm{x}$ & \\
\hline Alkan et al. (2017) & A & $\mathrm{X}$ & & $X$ & & & & & & & & \\
\hline Baldwin et al. (1991) & A & & & $\mathrm{X}$ & & & & & & & & \\
\hline Blattgerste et al. (2017) & C & $X$ & & & & & $\mathrm{X}$ & & & & & \\
\hline Boothroyd et al. (2011) & A & $\mathrm{X}$ & $X$ & & & & & & & & & \\
\hline Bäckstrand et al. (2008) & B & & & $\mathrm{X}$ & & & $\mathrm{X}$ & $\mathrm{X}$ & & & & \\
\hline Daniel \& Tversky (2012) & B & $\mathrm{X}$ & & & & & & & $\mathrm{X}$ & & & \\
\hline Falck et al. (2012) & A & & & & $\mathrm{X}$ & & & & & & & \\
\hline Falck et al. (2017) & A & & & & $\mathrm{x}$ & & & & & & & \\
\hline Funk et al. (2016) & C & & & & & & & & & & $\mathrm{X}$ & $\mathrm{X}$ \\
\hline Funk et al. (2017) & C & $\mathrm{X}$ & & & & & $\mathrm{x}$ & & & $x$ & & $\mathrm{X}$ \\
\hline Galy et al. (2012) & B & & & & & & $\mathrm{x}$ & $\mathrm{X}$ & & & & \\
\hline Ganier (2004) & B & & & & & & & & & $\mathrm{X}$ & & \\
\hline Heiser et al. (2004) & B & & & & & $\mathrm{X}$ & $\mathrm{X}$ & & $\mathrm{X}$ & & $\mathrm{X}$ & \\
\hline Kaipa et al. (2012) & B & & & & & $\mathrm{x}$ & & & & & & \\
\hline Lampen et al. (2019) & C & & & & & & $\mathrm{X}$ & $\mathrm{X}$ & & & & \\
\hline Lim \& Hoffmann (2015) & B & & & $\mathrm{X}$ & & & $\mathrm{x}$ & & $\mathrm{X}$ & $\mathrm{X}$ & & \\
\hline Menn et al. (2015) & B & & & & & & & $\mathrm{X}$ & & $\mathrm{X}$ & $\mathrm{X}$ & \\
\hline Mijksenaar \& Westendorp (1999) & B & & & & & $\mathrm{X}$ & $\mathrm{X}$ & & & & & \\
\hline Novick et al. (2000) & B & & & & & & & $\mathrm{X}$ & $\mathrm{X}$ & & & \\
\hline Otto (2003) & A & $\mathrm{X}$ & $\mathrm{X}$ & & & & & & & & & \\
\hline Sääski et al. (2008) & C & $\mathrm{X}$ & & & & & & & & & & $\mathrm{X}$ \\
\hline Sinha (2014) & A & & & $\mathrm{X}$ & & & & & & & & \\
\hline Söderberg et al. (2012) & B & & & & & & $\mathrm{X}$ & & $\mathrm{X}$ & & & $\mathrm{X}$ \\
\hline Syberfeldt et al. (2012) & C & & & & & & & & & & $\mathrm{x}$ & $\mathrm{X}$ \\
\hline Tversky \& Hemenway (1984) & B & & & & & & & & $\mathrm{X}$ & & & \\
\hline Tversky et al. (2008) & B & & & & & & & & & & & \\
\hline Ullman (2016) & A & $\mathrm{X}$ & $\mathrm{X}$ & & & & & & & & & \\
\hline Zaeh et al. (2009) & A & & $x$ & & $\mathrm{X}$ & $\mathrm{x}$ & $\mathrm{X}$ & & $\mathrm{x}$ & & & \\
\hline Zheng et al. (2015) & C & $\mathrm{X}$ & & & & & & & & & & $\mathrm{X}$ \\
\hline Zhu et al. (2008) & A & & & & $\mathrm{X}$ & & & & & & & \\
\hline
\end{tabular}

An interesting insight that can be gained from table 1 is that not much overlap exists between where multiple themes occur in one and the same article. In addition to that, finding number 6 regarding decoding of instructions and mental workload is well represented, which was expected.

\section{Conclusions}

Assembly operations are generally divided into: handling a part, and joining a part. Instructions should support the assembly worker by informing about four major aspects: when and what should be assembled as well as where and how the components should be assembled. Each of these aspects has an impact on the overall complexity of the assembly process. To realise which of these aspects is most critical for a specific 
assembly task and assembly worker (i.e. taking into account both the requirements of the task and the capabilities/limitations of the worker) can be of help in creating and delivering effective, tailor made instructions.

The main finding is that there is no one size fits all solution when it comes to the effectiveness of instructions, but that the type of instructions and the way they are delivered should be adjusted to the complexity of the assembly operations as well as adjusted to the experience of the worker, where possible. The outcomes mentioned in section 4.1 are seen as a basis for future work on generating for heuristics and guidelines when it comes to manual assembly instructions and what factors to consider. Testing the results of the literature study was planned, but has been delayed due to current conditions, and was planned as an experiment on assembly workers of varying experience levels, showing at least 2 different types of assembly instructions for tasks of varying complexity.

\section{Acknowledgements}

This work was supported by the TACO project (insTruction innovAtion for Cognitive Optimisation), funded by the Swedish innovation agency, Vinnova.

\section{References}

1.Zhu X, Hu SJ, Koren Y, Marin SP. Modeling of manufacturing complexity in mixed-model assembly lines. In: International Manufacturing Science and Engineering Conference (2006), 649-659.

2.Romero D, Stahre J, Wuest T, Noran O, Bernus P, Fast-Berglund $\AA$, Gorecky D. Towards an operator 4.0 typology: a human-centric perspective on the fourth industrial revolution technologies. In: Proceedings of the international conference on computers and industrial engineering (CIE46), Tianjin (2016) 29-31.

3.Mijksenaar, P., Westendorp, P. Open here: the art of instructional design. T\&H, London, (1999).

4.George ML, Maxey J, Rowlands DT, Upton M. Lean six sigma pocket toolbook. McGraw-Hill Professional Publishing (2004).

5.Menn JP, Müller BC, Seliger G, Ulbrich C. Generic model for international assembly instructions for special machinery assembly. In: Proceedings of 43rd Annual SEFI Conference, Orléans (2015).

6.Söderberg C, Johansson A, Mattsson S. Design of simple guidelines to improve assembly instructions and operator performance. In: The sixth Swedish Production Symposium (2014).

7.Wohlin C. Guidelines for snowballing in systematic literature studies and a replication in software engineering. In: Proceedings of the 18th international conference on evaluation and assessment in software engineering (2014), 1-10.

8.Li D, Cassidy T, Bromilow D. The Design of Product Instructions. Leeds: licensee InTech. (2013), 101-14.

9.Alkan B, Vera D, Ahmad B, Harrison R. A method to assess assembly complexity of industrial products in early design phase. IEEE Access (2017), 989-999.

10.Funk M, Kosch T, Schmidt A. Interactive worker assistance: comparing the effects of in-situ projection, head-mounted displays, tablet, and paper instructions. In: Proceedings of the 2016 ACM International Joint Conference on Pervasive and Ubiquitous Computing (2016) 934-939.

11.Blattgerste J, Strenge B, Renner P, Pfeiffer T, Essig K. Comparing conventional and augmented reality instructions for manual assembly tasks. In: Proceedings of the 10th international conference on pervasive technologies related to assistive environments (2017), 75-82.

12.Agrawala M, Phan D, Heiser J, Haymaker J, Klingner J, Hanrahan P, Tversky B. Designing effective stepby-step assembly instructions. ACM Transactions on Graphics (TOG). (2003, 828-837.

13.Tversky B, Agrawala M, Heiser J, Lee P, Hanrahan P, Phan D, Stolte C, Daniel MP. Cognitive design principles for automated generation of visualizations. Applied spatial cognition: From research to cognitive technology (2006), 53-75.

14.Boothroyd, G., Dewhurst, P. and Knight, W.A., Product Design for Manufacture and Assembly. CRC Press. 2010. 